Bullefin of the Seismological Society of America. Vol. 59, No. 6, pp. 2185-2196. December, 1969

\title{
TRANSIENT AND RESIDUAL STRAINS FROM LARGE UNDERGROUND EXPLOSIONS
}

\author{
By Stewart W. Smith, Charles B. Archambeau and William Gile
}

\section{ABSTRACT}

Tectonic strain readjustments associated with large underground explosions have been observed at the Nevada Test Site. The BENHAM event of December 19, 1968 produced a peak quasi-static radial strain of $1.2 \times 10^{-7}$ at a distance of $29 \mathrm{~km}$. This strain transient was followed by an exponential return to the initial state with a time constant of 13 minutes, and is interpreted as the direct elastic response of the medium to a time varying pressure in the BENHAM cavity. An upper bound on the tectonic strain release was determined to be $0.7 \times 10^{-8}$. Using these measurements it is estimated that the permanent and quasi-static strains associated with this explosion could significantly effect local earthquake occurrences out to distances of about $15 \mathrm{~km}$. The size distribution of aftershocks of this explosion resembles that seen in model experiments of brittle fracture, in which the distribution is controlled by the dimensions of inhomogeneities in the medium.

\section{INTRODUCTION}

A sensitive strain seismograph was operated in a deep tunnel at a distance of $29 \mathrm{~km}$ from a large underground explosion at the Nevada Test Site. For the location of this station refer to Figure 1. A transient strain was recorded here, see Figures 2 and 3, that resembled a step function with an initial offset of $3.0 \times 10^{-8}$ corresponding to a radial compression of $1.2 \times 10^{-7}$, followed by an exponential return to the initial state with a time constant of 13 minutes. The entire strain seismograph system was carefully checked for linearity and hysteresis. Coupling to the earth was confirmed by the recording of solid earth tides of the appropriate amplitude and phase for this locality, as can be seen in Figure 4. As a result of these tests there is little doubt that the observed transient represents actual strain in the earth rather than an instrumental artifact.

The observed initial strain offset, a compression, corresponds to the elastic deformation one would expect at this distance due to an overpressure of several hundred bars in a spherical region of radius 300 meters (see Figure 5). This region may correspond to the linear zone around the cavity. The time constant for the return to the unstrained state roughly corresponds to the cooling and pressure drop that one might expect for the cavity of an explosion of this size. In addition, several individual collapse events were seen to produce small changes in strain in a direction corresponding to a pressure drop in the cavity.

The initial strain at a distance of $29 \mathrm{~km}$ is of the same order of magnitude as the solid earth tide and thus cannot be expected to have any effect on the seismicity at this distance. Assuming that strains of at least $10^{-6}$ are required to activate existing faults in this region, we can see from Figure 5 that the area in which the static effects of the cavity may be expected to have some effect on local earthquake occurrences lies within about $15 \mathrm{~km}$ of the source. This result is consistent with earlier work, Smith et al. (1969), which suggested that the aftershock region of a large explosion can be estimated by comparing it with an earthquake of equivalent surface wave magnitude.

After correcting for the earth tide, it is clear that no residual strain greater than 


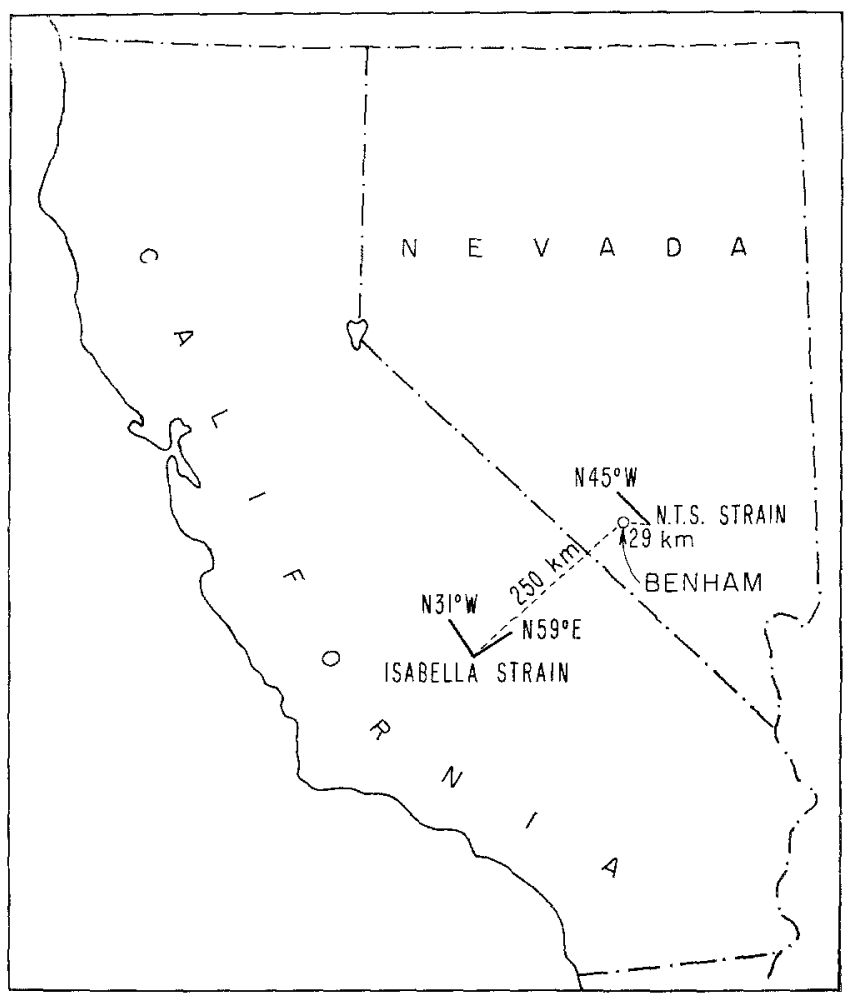

Fra. 1. Location and orientation of strain seismographs at Isabella and N.T.S. with respect to the Benham event.

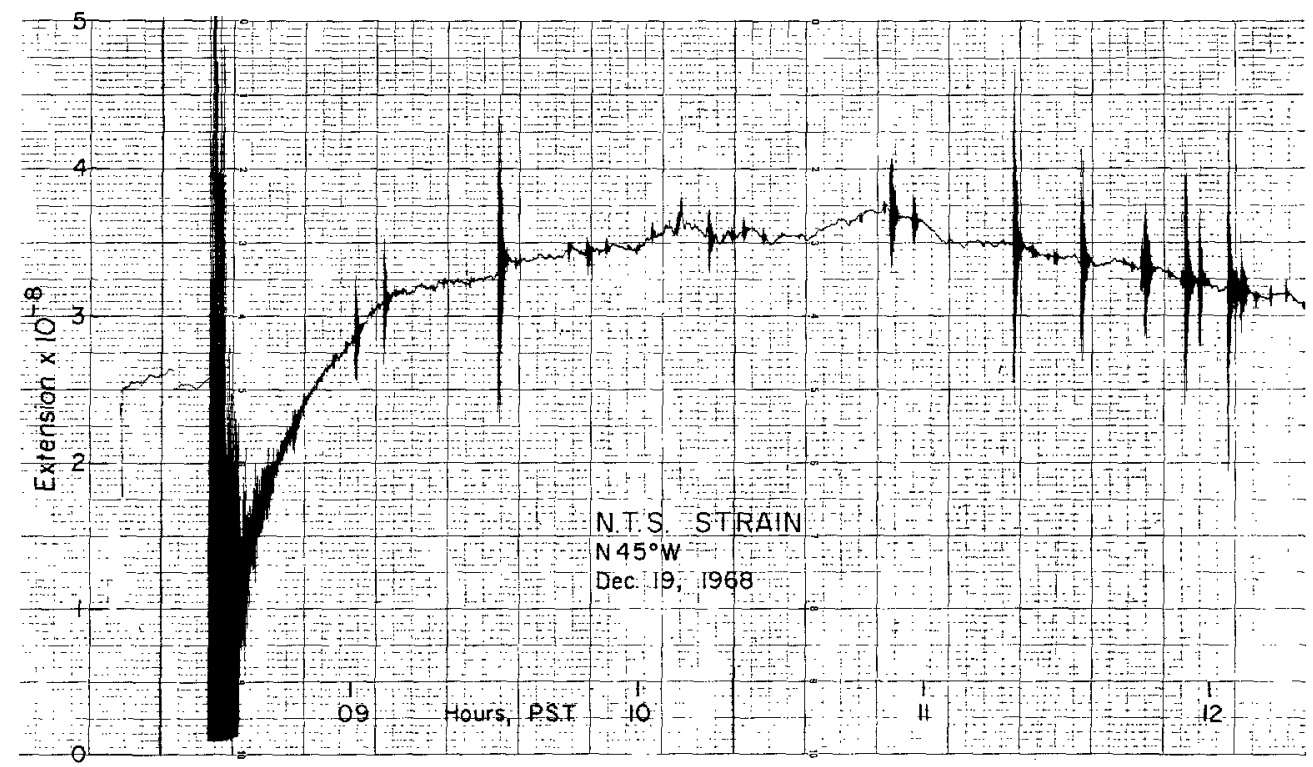

FIG. 2. High-gain recording of BENHAM event on N.T.S. strain seismograph at a distance of $29 \mathrm{~km}$. Initial quasi-static strain offset is $3.0 \times 10^{-8}$ followed by exponential recovery with time constant of 13 minutes. Maximum in earth tide occurs at 1030 P.S.T. 
$0.7 \times 10^{-8}$ could have occurred. This establishes an upper limit for the tectonic strain release for this particular location and direction of observation. Press and Archambeau (1963) calculated the relaxation effect of inserting a stress free cavity into a pre-stressed region. Using their result, and a $500 \mathrm{~m}$ radius as an upper bound for the crushed zone

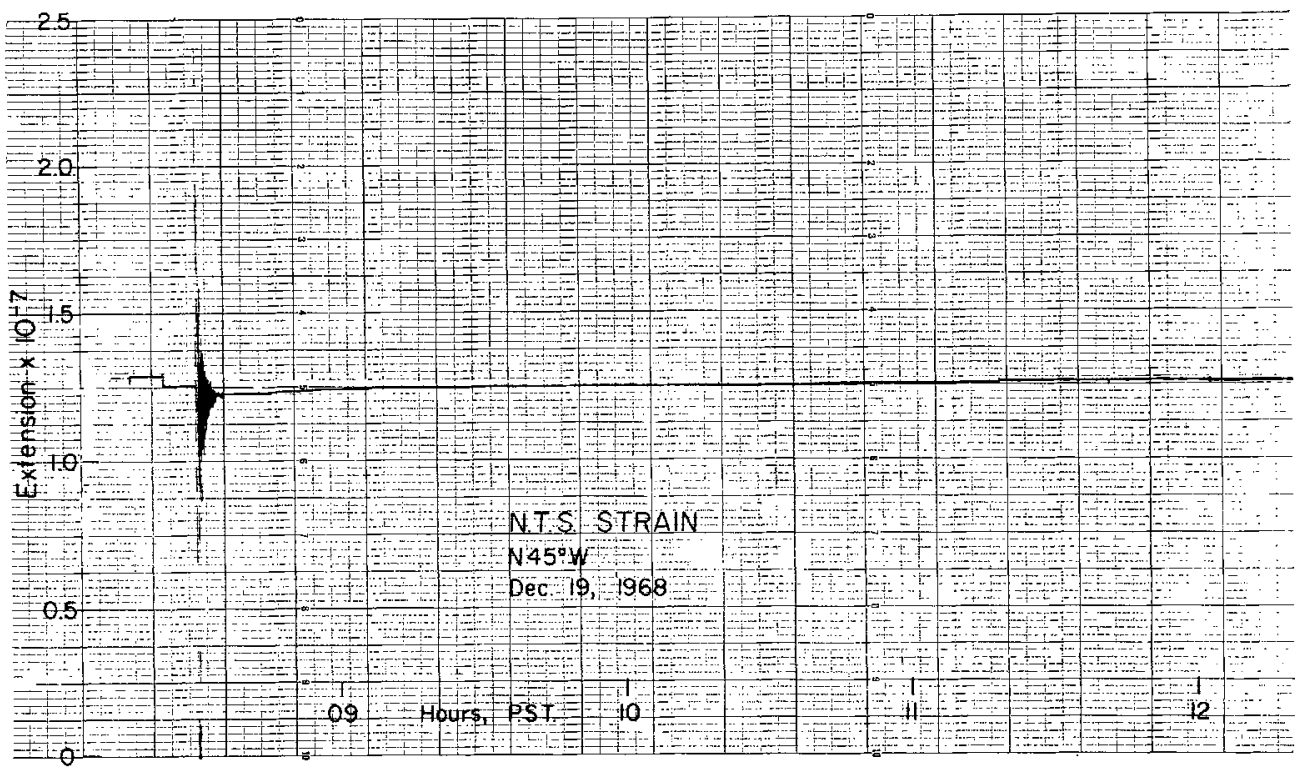

FIG. 3. Low-gain recording of BenHAM event on N.T.S. strain seismograph at a distance of $29 \mathrm{~km}$. Disturbance prior to explosion is recorder recentering.

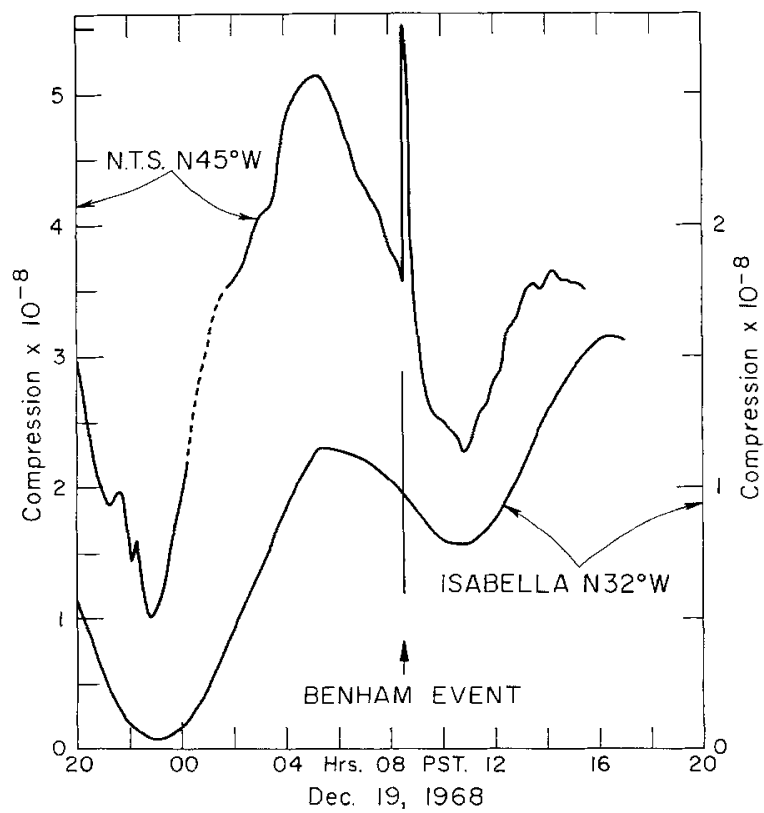

FIG. 4. Strain recordings smoothed and replotted on compressed time scale to show solid earth tide at Isabella and at N.T.S. Agreement between stations is considered good. Amplitude differences are due to difference in azimuth of strainmeters and geologic conditions at the two sites. 
obtained by scaling the observations of BruBr, Archambeau and Sammis (1969), we find that the theoretically expected tectonic release due to BENHAM is consistent with the observed upper bound as shown in Figure 5 .

At Isabella, California, a distance of $250 \mathrm{~km}$ to the southwest, transient strains were also measured for the Boxcar event, Smith et al. (1969), and the BenHam event. In neither case, were permanent or quasi-static strain changes discernible. Careful meas-

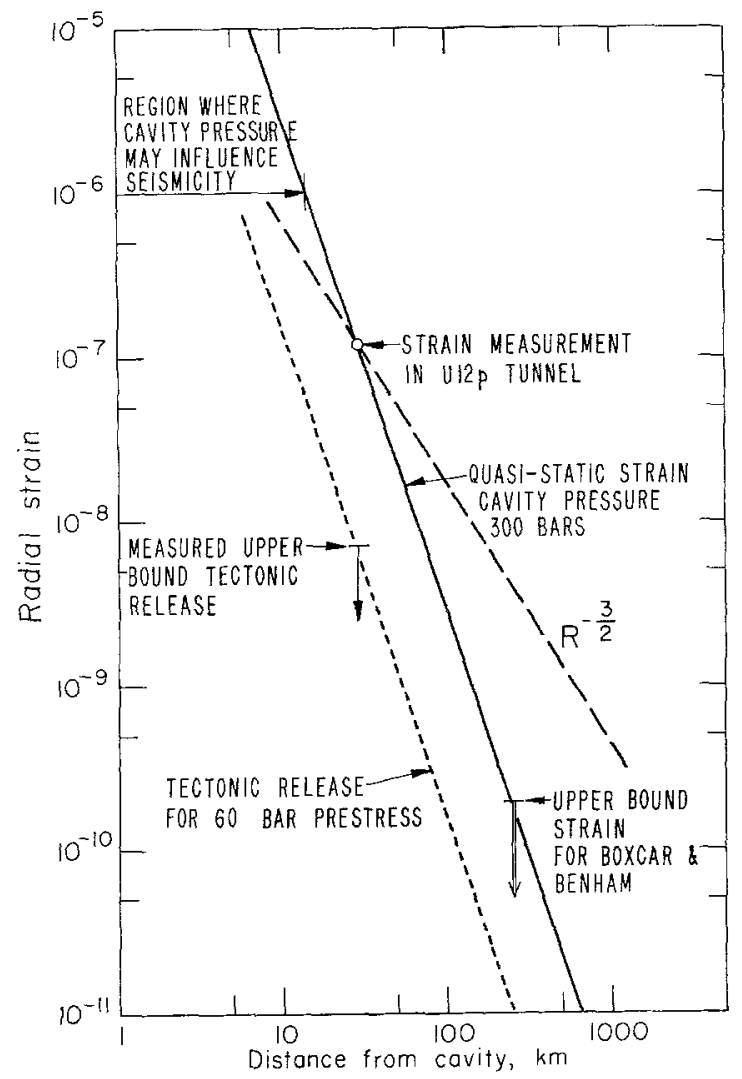

Fig. 5. Observed radial strain at Isabella and N.T.S. for BENHAM and BoxCAR events. Dashed line represents theoretically predicted tectonic strain release assuming a fractured zone radius of $450 \mathrm{~m}$ and a pre-stress of 60 bars. Solid line represents elastic strain produced by a cavity of radius $300 \mathrm{~m}$ (crushed zone) with an overpressure of 300 bars. Both theoretical curves have a distance dependence of $R^{-3}$. For comparison, a curve proportional to $R^{-3 / 2}$ is shown by a broken line.

urement of these records, with consideration for the earth tide, established an upper limit on strain changes with a duration greater than about 10 minutes to be $2.0 \times 10^{-10}$.

\section{Earthquake Strain Readjustment}

The Earth's crust is continually undergoing elastic and anelastic readjustment to accomodate the internal motions responsible for the evolution of this planet. These motions can most clearly be observed over long periods of time by means of geodetic techniques. The readjustments associated with large earthquakes can be measured directly by means of earth strain gages at distances of many hundreds of $\mathrm{km}$. Because such data have a wide scatter however, there are serious problems in its interpretation. Recently, McGinley (1968) made a detailed study of several clearly recorded examples of strain readjustments associated with earthquakes for which the focal mechanisms 
were well understood. He concluded that local perturbations in the strain field near the recording instrument must have played an important part in determining the total apparent strain change that accompanied these earthquakes. Such local effects would seem most likely to be caused by small motion along existing fractures induced by the dynamic loading of the seismic waves. The current experiments at the Nevada Test Site provide an opportunity to measure elastic readjustments in the crust due to the introduction of large cavities as well as to determine the local tcetonic readjustments that may be caused by dynamic loading. The observation of an initial compression associated with the BENHAM event followed by an exponential return to equilibrium we interpret to be the direct elastic effect of the pressure history in the BENHAM cavity a distance of $29 \mathrm{~km}$ away. The capability to see such elastic effects here clearly establishes this locality as an ideal one to observe the static effects of large earthquakes in the Western U.S. and Mexico.

\section{Strain Measurement}

On December 9, 1969 a conventional quartz strain seismograph was installed within the abandoned tunnel U12p at the Nevada Test Site. The tunnel is located at $37^{\circ} 13.8^{\prime} \mathrm{N}$ and $116^{\circ} 09.2^{\prime} \mathrm{W}$ at an elevation of 5500 feet. The instrument was installed $2000 \mathrm{ft}$. from the portal, the depth below the surface at this point is approximately $600 \mathrm{ft}$. Tunnel construction had been suspended in September 1962, and no use has been made of the tunnel since that time. As a result, the environment was in thermal equilibrium, and little disturbance was caused by the installation of the strain gage. The tunnel is located in vitric tuffs of Pliocene or younger age that strike generally $\mathrm{N}$ to NNW and dip $3^{\circ}$ to $10^{\circ} \mathrm{E}$. Bowers and Emerick (1963) mapped 16 normal faults in the tunnel with apparent displacements usually less than 2 inches, and described the distribution of joints. Comparing this tunnel with typical sites for strainmeters in tectonically active areas, it would be described as remarkably homogeneous and undeformed. The interior is extremely dry, and there is no evidence of entry of surface water beyond about $50 \mathrm{~m}$ of the portal. From the standpoint of homogeneity of rocks, temperature, and ground water circulation, the location seems an ideal one for measurement of tectonic strain.

To reduce the stabilization time for the installation, no concrete was used. A small flat surface was prepared on the welded tuff, and the instrument piers were bonded to it directly with epoxy. As a result, within about 72 hours the strainmeter had stabilized such that it could be operated with a full scale sensitivity of $5 \times 10^{-8}$. Although daily thermal fluctuations are larger than had been expected for this depth of burial, the semi-diurnal solid earth tide can be seen. This is illustrated in Figure 4 and compared with the observed tide at Isabella, California. The correlation is good, and confirms the fact that this new site is well coupled to the Earth's crust. Observation of the semidiurnal eath tide is perhaps the only adequate way of checking the performance of a sensitive earth strain gage. The output of the transducer was recorded on a small battery operated recorder in the tunnel, and also telemetered to Mercury, Nevada where it was recorded at two levels of sensitivity. All of the instrumentation was powered by solar panels located at the entrance to the tunnel.

\section{Static Effect of Cavity}

Since the explosive source region is at most a few hundred meters in dimension, and the strain measurements were made at a distance of almost $30 \mathrm{~km}$, a point source representation seems valid to investigate the relation between cavity pressure and. 
strain. Mindlin and Cheng (1950) give the displacement field in an elastic half space for a variety of point force situations. For a point source of dilatation they give

$$
U_{x}=\frac{X S}{2 \mu}\left[\frac{1}{R_{1}{ }^{3}}+\frac{3-4 \sigma}{R_{2}{ }^{3}}-\frac{6 Z(Z+C)}{R_{2}{ }^{5}}\right]
$$

where $S$ is the product of pressure and source volume, $R_{1}{ }^{2}=X^{2}+Y^{2}+(Z-C)^{2}$, $R_{2}^{2}=X^{2}+Y^{2}+(Z+C)^{2}$ and $C$ is the source depth. Since the depth of burial is small compared with the distance, we can let both the source and receiver depths be zero. To further simplify matters, we take Poisson's ratio $\sigma=\frac{1}{4}$. The strain $e_{x x}$ then becomes

$$
e_{x x}=\frac{3 S}{2 \mu}\left[\frac{R^{3}-3 X^{2} R}{R^{6}}\right]
$$

where $x$ is the distance along the surface, $R$ is the slant distance and $\mu$ is the rigidity. In the case at hand, the strain measured along a line N45W due to a source located a distance $R$ to the west is just

$$
e_{x x}=-\frac{\pi P}{\mu}\left(\frac{R_{c}}{R}\right)^{3}
$$

where $P$ is pressure, and $R_{c}$ is equivalent cavity radius.

Since the predicted strain effects depend on the cube of the cavity radius, it is of considerable importance to determine this number as accurately as possible. Using the experimental results of Boardman et al. (1964) and the following parameters: yield 1 megaton, density $1.85 \mathrm{gm} / \mathrm{cm}$, rigidity $3 \times 10^{10}$ dyne per $\mathrm{cm}^{2}$, and depth 4300 feet, we find the expected cavity radius to be 100 meters. If however a fractured zone exists around the cavity through which pressures can be freely communicated in the sense that the region behaves plastically or as a finely cracked material with very low rigidity, this larger zone will be the appropriate one to use in calculating the far field static elastic effects of the cavity. To further complicate the picture, we note that the even larger zone of radial cracking and joint opening will serve as the effective radius for tectonic stress relaxation. Since measurements of these three characteristic cavity radii are not available, we will build a self-consistent argument in the following way. Using the measured radial strain reported in this paper and assuming that the cavity pressure at the end of the cavity formation period did not exceed twice the lithostatic pressure, we find from equation (1) that the appropriate cavity radius for far-field elastic effects is $300 \mathrm{~m}$. Clearly this will also be a lower bound on the effective radius for tectonic stress release. Archambeau and Sammis (1969) determined the effective stress release zone for the BILBy event, Scaling their result to the present case we obtain a radius of $500 \mathrm{~m}$. The expected tectonic strain release calculated from these two values brackets the observed upper bound.

Results of the foregoing discussion are summarized in Figure 5 in which we see that calculations of both tectonic strain release and direct static effects of cavity pressure are consistent with experimental observations. For comparison we show on the same figure a strain curve proportional to $R^{-3 / 2}$ as has been suggested by Wideman and Major (1967). This curve predicts a quasi-static strain at Isabella more than one order of magnitude larger than the experimentally determined upper bound determined 
here for both the BoxcaR and BeNHAM events. It is possible that previous observations of a strain-distance variation of $R^{-3 / 2}$ are a result of permanent strain changes near the recording site induced by the dynamic overloading of near surface fractures that occurs with the passage of large amplitude surface waves. Such a phenomenon would be expected to show the same velocity as surface waves, and to have an amplitude-distance relationship of $R^{-3 / 2}$.

\section{StRain Recovery}

Since the large transient strain produced by BENHAvr is in the same direction as the first motion, that is a compression, it could have been caused by either tectonic release in the surrounding medium, or a net overpressure in the cavity. Calculations of expected tectonic effects discussed previously showed them to be several orders of magnitude smaller than the observed transient effect, thus we conclude that the transient is a direct mechanical effect of the cavity. As such, the exponential return to equilibrium can be explained as either the pressure drop in the cavity due to cooling, or the anelastic stress relaxation of material near the cavity. Without more information on the pressures and temperatures involved, it is not possible to distinguish between these two hypotheses. Howrever a plausibility argument based on scaled results from smaller explosions leads us to prefer the explanation that the 13 minute decay of strain is a direct elastic effect of the pressure drop in the cavity.

Olsen (1967) calculated the pressure history for a nuclear detonation in alluvium and compared it with experimentally measured values. In his model, the final pressure after cavity formation was assumed to be equal to the overburden pressure, and this assumption was confirmed by experiment. The subsequent pressure history was controlled by cooling and condensation of water vapor within the cavity, until collapse occurred. There are several important differences between the situation described by Olsen and that discussed here. The yield which was much larger in the present case, which would produce significantly higher temperatures, and the material, a welded tuff, had considerably more strength than alluvium. For these two reasons, one might expect significant differences in the pressure history. The depth of burial, 4300 feet, and the rigidity of the material $3 \times 10^{10}$ dyne $/ \mathrm{cm}^{2}$ leads us to believe that the final pressure after cavity formation could have been significantly above the lithostatic pressure. Olsen, (personal communication) also indicated that overpressures of several times the lithostatic pressure seem reasonable in light of both theoretical and experimental work on large explosions. Using the observed peak quasi-static strain reported in the present paper we calculated that the over-pressure at the time of cavity formation would be between 300 and 1000 bars if the cavity radius was between 200 and $300 \mathrm{~m}$. Since the lithostatic pressure was approximately 300 bars, these values do not seem unreasonable. We note that a complicated elastic "doming" effect should have occurred at the surface within several $\mathrm{km}$ of the cavity and that this elastic deformation would be fully recovered within about 1 hour of the explosion.

After formation of the cavity, the pressure will return to approximately the original lithostatic value, through various processes of cooling and stress relaxation in the surrounding medium. The cavity of course will not disappear at this time because it is formed principally by the non-linear compression of materials, deformation that is not recovered after the pressure returns to equilibrium. The time constant for the decay of pressure will depend on the cavity volume, the amount of water vapor, the temperature, and the heat loss through the cavity walls. Olsen (1967) assuming Newtonian cooling of the cavity in which the material behaved as a perfect gas, expressed the 
temperature and pressure history as proportional to exp (- heat loss rate/total heat capacity) time. Although it is not possible to construct a simple scaling relation for the pressure history, we note that the total heat capacity depends on the cavity volume and its contents, and thus is a function of yield. For large yields one expects in general larger time constants. If heat capacity were simply proportional to volume, the time constant would scale linearly with cavity radius, and a 13 minute decay might be expected to be characteristic of a 50 meter cavity. Since the actual cavity was probably close to 100 meter, we can at least say that it is plausible that the observed strain decay is caused by the cooling history and pressure drop in the cavity.

\section{Tectonic Strain Release}

As mentioned earlier, in the zone around the cavity in which there is a high density of radial cracks and opening of pre-existing cracks and joints, one would expect a rather complete relaxation of the existing tectonic pre-stress. The previous arguments on cavity size established bounds on the radius of this region to be between $300 \mathrm{~m}$ and $500 \mathrm{~m}$. Press and Archambeau (1963) determined that the insertion of a spherical fracture zone in a pre-stressed region would produce a strain change

$$
e_{i j}=\frac{15(\lambda+\mu) S_{12}}{9 \lambda+14 \mu}\left[P_{i j}(\theta, \phi)\left(\frac{R_{0}}{R}\right)^{j}-Q_{i j}(\theta, \phi)\left(\frac{R_{0}}{R}\right)^{3}\right]
$$

where $P_{i j}$ and $Q_{i j}$ are only functions of the angles. In the case at hand, $\theta=90^{\circ}, \phi=45^{\circ}$, $R=29 \mathrm{~km}$ and we obtain

$$
e_{x x}=-1.14 S_{12}\left(\frac{R_{0}}{R}\right)^{3}+.31 S_{12}\left(\frac{R_{0}}{R}\right)^{5}
$$

where $S_{12}$ is the pre-strain. Archambeau and Sammis (1969) estimate the pre-stress in this region to be 75 bars. For tuff with a rigidity of $3 \times 10^{10}$ dyne per $\mathrm{cm}^{2}$ this is equivalent to a strain of $2.5 \times 10^{-3}$. This value is quite large and we view it as an upper limit for acceptable strain due to pre-stress. A calculation of the observed strain using eq. (2) and values for $S_{12}$ and $R_{0}$ based on the above estimates is plotted in Figure 5 in which we see that a pre-stress of 60 bars and a fracture zone radius of $450 \mathrm{~m}$ produces a tectonic release just equal to the observed upper bound at the NTS station site.

Another important estimate is the total energy to be associated with the permanent strain changes due to stress relaxation around the rupture zone. We have for the energy reduction and released as seismic radiation, Archambeau and Sammis (1969)

$$
E_{R}=\frac{1}{2} \mu S_{12}^{2}\left(\frac{4}{3} \pi R_{0}^{3}\right)\left[f\left(\frac{\mu}{\lambda}\right)+\left(1-\left(\frac{R_{c}}{R_{0}}\right)^{3}\right)\right]
$$

where

$R_{c}=$ crushed zone radius (corresponds to the non-linear zone of $300 \mathrm{~m}$. obtained for BENHAM previously)

$R_{0}=$ outer radius of the fracture zone, including the zone of radial fracture (corresponds to the fracture zone radius of $450 \mathrm{~m}$. obtained for BENHAM) 


$$
f\left(\frac{\mu}{\lambda}\right) \simeq 1 \text { for tuff. }
$$

With the parameters appropriate for the present case we have

$$
E_{R}=3.65 \times 10^{19} \text { ergs. }
$$

The energy is released primarily as $S V$ and $S H$ seismic waves. This energy corresponds to the energy released seismically in a magnitude 5.7 event (unified magnitude, $m$, where $\log E=5.8+2.4 \mathrm{~m}$, Gutenberg and Richter, 1956).

\section{EARThQUAKE Triggering}

It is of considerable interest to estimate the effects of subjecting a tectonically active region to the kinds of stresses described earlier in this paper. Over what region might one expect the static overloading of the source cavity to have a significant effect on local seismicity? As a lower bound, we may safely say that strains of the order of $10^{-7}$ to $10^{-8}$ can have little or no effect, since the crust is continually subjected to such deformation due to the solid earth tides. As an upper bound we note that a strain of the order of $10^{-3}$ to $10^{-4}$ is observed for this tectonically active region, at least in the Tuff (Archambeau and Sammis, 1969). Thus a strain of $10^{-3}$ would be expected to cause an earthquake even without the existence of a fault or of a prestress. A much smaller strain might be effective in the case of a prestressed medium, and particularly when a fault zone or zone of weakness concentrates the stress. As a lower limit on the strain necessary to activate a fault in this region we would therefore estimate a value of $10^{-6}$. This is consistent with observed strains associated with earthquakes in California and is at least an order of magnitude higher than solid earth tide strains. Figure 5 shows that strains of this size can be expected at distances of less than $15 \mathrm{~km}$.

It is important not to lose sight of the fact that the above discussion applies only to the static or quasi-static effect of the cavity. The dynamic loading produced by the seismic waves may in fact be the most important factor in triggering nearby earthquakes. In this sense, an explosion might be viewed as a "foreshock." From the low gain record in Figure 3 we see that the dynamic strains at a distance of $29 \mathrm{~km}$ exceeded $2.0 \times 10^{-7}$. In the near field region such strains also will have an $R^{-3}$ dependence on distance. Thus one can expect very high strain levels in the region close to the source. No comment can be made about the effect of such waves on earthquake occurrences other than to point out that they may be of equal or greater importance than the static effects which are the principal concern of this paper.

\section{AFTERSHOCKS}

In the 23 hour interval immediately following the BenHam event 82 aftershocks were clearly recorded on the strain seismograph at N.T.S. The time of occurrence and peak strain amplitude are listed in Table 1. The minimum discernible peak dynamic strain corresponding to $3 \mathrm{~mm}$ on the record charts was $0.6 \times 10^{-9}$. Peak frequencies were estimated to be $20 \mathrm{cps}$. Assuming all events had a similar frequency content and occurred close to the source thus being at the same distance from the strainmeter, the peak dynamic strains are a direct measure of the relative energy or magnitude of these aftershocks. Some of these events were undoubtedly collapse type features, occurring at the boundaries of the cavity. Others were true aftershocks in the sense that they were readjustments to the strain field that was perturbed by the explosion. As such, it is valid to compare their statistics with those of earthquake aftershocks. The plot shown in 
Figure 6 illustrates the distribution of the number of events versus their relative energy or magnitude. Although the sequence was short, with only 82 events, it appears to

TABLE 1

Time of Occurrence and Maximum Peak to Peak Strain for Afterheocks of THE BENHAM EVENT

\begin{tabular}{|c|c|c|c|}
\hline Time P.S.T. & Strain & Time P.S.T. & Strain \\
\hline Dec. 19 & & 29.7 & 6 \\
\hline 0847.5 & $35 \times 10^{-10}$ & 31.0 & 24 \\
\hline 55.9 & 9 & 35.8 & 13 \\
\hline 58.3 & 9 & 40.6 & 180 \\
\hline 59.7 & 6 & 48.1 & 53 \\
\hline 0900.4 & 84 & 56.2 & 6 \\
\hline 03.6 & 11 & 1310.3 & 11 \\
\hline 06.3 & 55 & 13.1 & 9 \\
\hline 18.8 & 9 & 14.5 & 130 \\
\hline 30.1 & 250 & 34.0 & 12 \\
\hline 34.6 & 11 & 55.0 & 13 \\
\hline 44.9 & 15 & 1403.4 & 15 \\
\hline 49.0 & 31 & 05.2 & 200 \\
\hline 52.7 & 15 & 07.1 & 57 \\
\hline 1002.7 & 17 & 12.1 & 66 \\
\hline 07.7 & 15 & 14.4 & 13 \\
\hline 14.5 & 48 & 17.2 & 64 \\
\hline 19.1 & 13 & 20.7 & 97 \\
\hline 21.5 & 20 & 23.2 & $700+$ \\
\hline 25.6 & 13 & 28.7 & 81 \\
\hline 46.3 & 7 & 1515.1 & 42 \\
\hline 51.0 & $\begin{array}{r}9 \\
84\end{array}$ & \multicolumn{2}{|c|}{ Record speed change } \\
\hline $\begin{array}{l}52.4 \\
57.3\end{array}$ & 46 & 1616 & 22 \\
\hline 1118.3 & 320 & 1708 & 59 \\
\hline 22.1 & 13 & 1721 & 24 \\
\hline 26.8 & 11 & 1743 & 75 \\
\hline 32.5 & 160 & 1823 & 33 \\
\hline 43.0 & 9 & 1930 & 6 \\
\hline 44.9 & 42 & 2013 & $18 \times 10^{-10}$ \\
\hline 45.8 & 100 & 2101 & 11 \\
\hline 50.7 & 9 & 2132 & $110 \pm$ \\
\hline 53.3 & 15 & 2330 & 6 \\
\hline 54.3 & 180 & Dec. 20 & \\
\hline 57.1 & 100 & 0019 & 6 \\
\hline 1158.7 & $280 \times 10^{-10}$ & 0044 & $18 \pm$ \\
\hline 1205.8 & 75 & 0150 & 40 \\
\hline 08.9 & 13 & 0156 & 90 \\
\hline 11.9 & 15 & 0159 & 7 \\
\hline 15.3 & 20 & 0201 & 27 \\
\hline 18.8 & 6 & 0348 & 8 \\
\hline 20.6 & 15 & 0407 & 11 \\
\hline 21.1 & 24 & 0819 & 42 \\
\hline
\end{tabular}

deviate significantly from the power function distribution one would expect for earthquake aftershocks. It appears to flatten out for events with peak strain amplitudes of less than $0.6 \times 10^{-8}$. This flattening of the magnitude frequency curve for small events has been observed in the laboratory, Mogi (1962) and attributed to the characteristic 
length of fractures in the material. This characteristic length is in turn controlled by the inhomogeneities of the medium. In the case of the BenHAm aftershock sequence the characteristic length was probably controlled by the existing fractures on which motion was induced during the initial disturbance. The determination of the characteristic length will require the actual aftershock magnitudes which have not yet been calculated from the present data. Because the data cannot be represented as a simple power function it is not possible to make a direct comparison with typical earthquake magnitude-frequency curves to determine if this region is one of high or low stress.

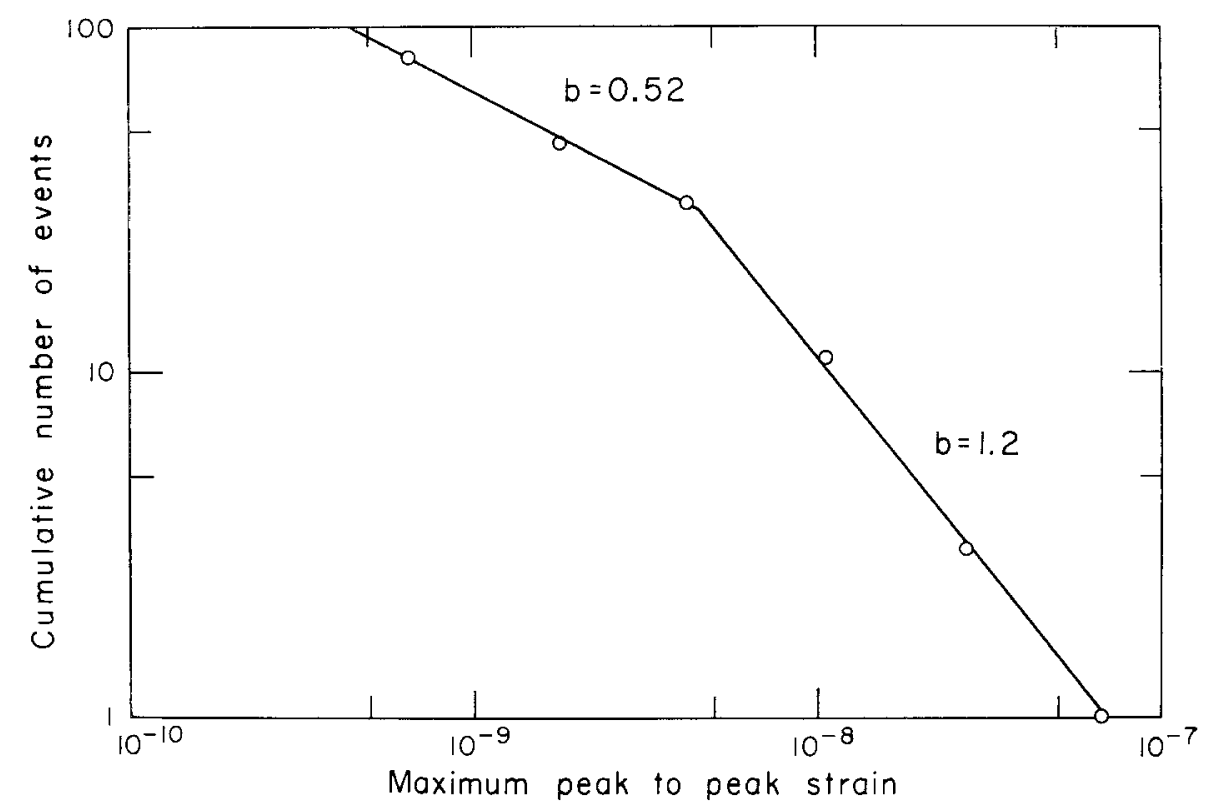

FIG. 6. Distribution of number of events versus relative energy for BENHAM aftershocks recorded by N.T.S. strain seismograph at a distance of $29 \mathrm{~km}$.

\section{ACKNOWLEDGMENTS}

This research was supported by the Effects Evaluation Division, Nevada Operations Office' U.S.A.E.C., and by the Air Force Office of Scientific Research under contract AF-AFOSR-62-421 Installation and operation of the equipment would not have been possible without the generous assistance of the U. S. Geological Survey personnel at Mercury, Nevada. The Jet Propulsion Laboratory made available the solar panels necessary for power at this remote location. We would also like to acknowledge the cooperation and assistance we received from many individuals associated with the Nevada Operations.

\section{ReFERENCES}

Archambeau, C., and C. Sammis (1969). Seismic radiation from explosions in prestressed media and the measurement of tectonic stress in the earth, Rev. Geophys., in press.

Boardman, C. R., D. D. Rabb, and R. D. McArthur (1964). Responses of four rock mediums to contained nuclear explosions, J. Geophys. Res. 69, 3457-3469.

Bowers, W. E., and W. L. Emerick (1963). Preliminary report on the geology of the U12p tunnel, Nevada Test Site, Nye County, Nevada, U. S. Geological Survey Technical Letter, Area 12-6.

Gutenbers, B., and C. F. Richter (1956). Magnitude and energy of earthquakes, Ann. Geofisica 9, 1-15.

Olsen, C. W. (1967). Time history of the cavity pressure and temperature following a nuclear detonation in alluvium, $J$. Geophys. Res. 72, 5037-5041. 
Press, F., and C. Archambeau (1963). Release of tectonic strain by underground nuclear explosions, J. Geophys. Res. 67, 337-342.

McGinley, J. R. (1968). A comparison of observed permanent tilts and strains due to earthquakes with those calculated from displacement dislocations in elastic earth models, Ph.D. Thesis, Calif. Inst. of Tech.

Mindlin, R. D., and D. H. Cheng (1950). Nuclei of strain in the semi-infinite solid, J. of Appl. Physics 21, 926-930.

Mogi, K. (1962). Magnitude-frequency relation for elastic shocks accompanying fractures of various materials and some related problems in earthquakes, Bull. Earihquake Res. Inst. 40, 831853.

Smith, S., J. McGinley, C. Scholz, and L. Johnson (1969). The effects of large explosions on tectonic strain, J. Geophys. Res., 74, 3308-3309.

Wideman, C. W., and Major, M. W. (1967). Strain steps associated with earthquakes, Bull. Seism. Soc. Am. 57, 1429-1444.

Division of Geologrcal Sciences

California Institute of Technology

Pasadena, California

Contribution No. 1614

Manuscript received June 14, 1969. 